\title{
Composición química y degradación ruminal de la vaina de mezquite (Prosopis spp.) a diferente estado de madurez
}

\section{Chemical composition and ruminal degradation of the mesquite pod (Prosopis spp.) at different maturity stages}

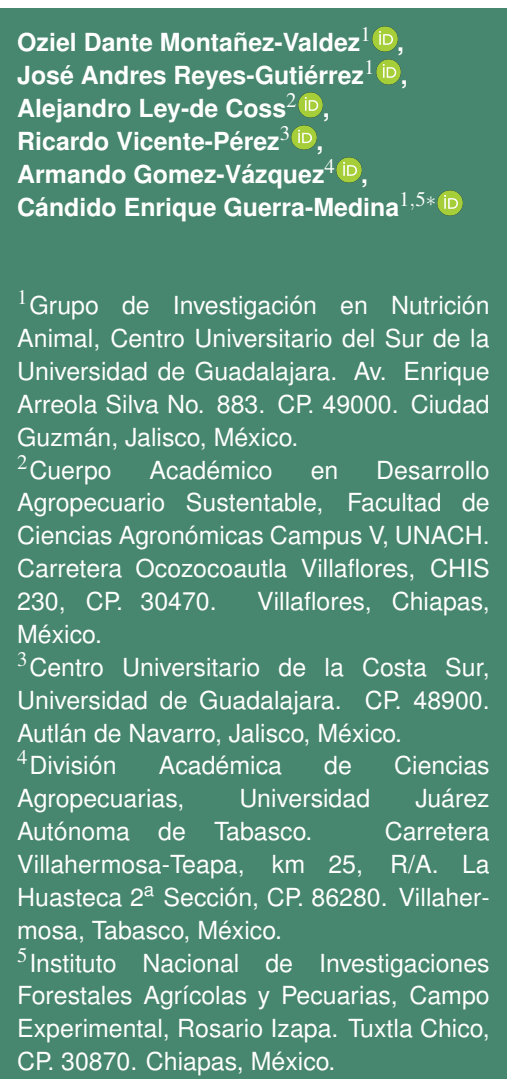

CP. 30870. Chiapas, México.

*Autor de correspondencia:

eguerranutricion@gmail.com

Artículo científico

Recibido: 11 de febrero 2021

Aceptado: 10 de septiembre 2021

Como citar: Montañez-Valdez OD, ReyesGutiérrez JA, Ley-de Coss A, VicentePérez R, Gomez-Vázquez A, GuerraMedina CE (2021) Composición química y degradación ruminal de la vaina de mezquite (Prosopis spp.) a diferente estado de madurez. Ecosistemas y Recursos Agropecuarios Núm. Esp. II: e2857. DOI: 10.19136/era.a8nll.2857
RESUMEN. El objetivo del estudio fue evaluar la composición química y la degradabilidad in situ (DIS) de la materia seca (MS) y orgánica (MO) de la vaina de mezquite a distinto estado de madurez en la localidad de Usmajac del municipio de Sayula, Jalisco, México. Los tratamientos evaluados fueron: a) vaina tierna (VT); de coloración verde fuerte y con semillas aun en formación; b) vaina madura (VM); de coloración morado intenso con líneas verdes y textura blanda; y c) vaina seca (VS); coloración morado pálido, con las semillas completamente formadas, una vaina gruesa y textura dura. La composición química de las vainas fue evaluada; para la DIS se utilizaron dos novillos Holstein con cánula ruminal permanente para incubar por cuadriplicado cada uno de los tratamientos experimentales por 16, 24, 36, 48, 72, $96 \mathrm{~h}$. VT, tuvo el mayor contenido de proteína cruda, VM y VS presentaron mayor contenido de carbohidratos no fibrosos. Se encontraron diferencias $(P<$ 0.05 ) entre tratamientos en la DIS de la MS, las vainas maduras tuvieron los valores más altos. Hubo diferencia entre tratamientos $(P<0.05)$ en la DIS de la MO, hasta las primeras $36 \mathrm{~h}$, VT y VM mostraron los valores más altos, posterior a esta hora no hubo diferencia entre ellos $(P>0.05)$. De acuerdo con la composición química y la digestibilidad in situ de la vaina de mezquite, puede considerarse como una alternativa para la alimentación de rumiantes.

Palabras clave: Alimentación, bovinos, estiaje, leguminosas, nutrición.

ABSTRACT. The aim of this study was to evaluate the chemical composition and in situ degradability (DIS) of the dry matter (DM) and organic matter (OM) matter of the pods from Mesquite (Prosopis spp.) at different maturity stage in the location of Usmajac, Sayula County, Jalisco, Mexico. The evaluated treatments were: a) Tender pods (VT); strong green color with seeds still in formation; b) Mature pods (VM); intense purple with green lines and soft texture; and c) Dry pod (VS); pale purple color and with fully formed seeds, thick pods with a hard texture. The chemical composition of the pods was evaluated. The DIS was calculated using two Holstein steers with permanent ruminal cannula, quadruplicate samples of each experimental treatments were incubated for $16,24,36,48,72,96 \mathrm{~h}$. Tender pod, had the highest content of crude protein, VM and VS had the highest content of non-fibrous carbohydrates. Differences were found between treatments in the DIS of the DM $(P \leq 0.05)$, the mature pods showed the highest values. There was a significative difference between treatments in the DIS of the OM $(P<0.05)$, VT and VM showed the highest values at $36 \mathrm{~h}$ incubation period. Incubation time from 48 to $96 \mathrm{~h}$ showed no difference $(\mathrm{P}>0.05)$. According to the chemical composition and the in situ degradability, it is concluded that the mesquite pods can be considered as an alternative feed resource for ruminants.

Key words: Feeding, cattle, dry season, legumes, nutrition. 


\section{INTRODUCCIÓN}

En México, se han utilizado diversos tipos de árboles en los sistemas de producción pecuaria, en los que destacan los árboles del género Prosopis spp., Leucaena spp. y Acacia spp., estas especies son valoradas por su capacidad de enriquecimiento del suelo, mantener la cubierta vegetal en los desiertos o tierras con baja fertilidad, la obtención madera, usos medicinales y forraje (Xu et al. 2006, Gómez et al. 2009). El Prosopis spp. conocido comúnmente como mezquite, crece en zonas con escasa precipitación, suelos arenosos, terrenos salinos y pedregosos no aptos para el cultivo. En México, se puede encontrar mezquite en casi toda la república dado que más del $40 \%$ de su territorio son zonas áridas y semiáridas, ideales para su crecimiento (Chatuverdi y Sahoo 2013, Carrillo et al. 2014, Armijo et al. 2019), que puede ser considerado como una alternativa para la alimentación de los rumiantes. Esta especie también es abundante en Estados Unidos, Perú, Chile, Argentina, Brasil, Australia, Haití, Paquistán y en las partes áridas de la India (López et al. 2006). El ganado no consume ramas u hojas, pero las vainas producidas dos veces al año, se han utilizado como suplemento para el ganado (Andrade et al. 2011, Ríos et al. 2012). Por su alto contenido de proteína y carbohidratos solubles (Tawel et al. 2005), también se ha utilizado en dietas integrales para bovinos, ovejas, camellos, búfalos, conejos, aves de corral y ratas, en regiones de América del Sur, África y la India (Sawal et al. 2004, Khobondo 2019). Sin embargo, el valor nutricional de la vaina puede variar según el estado de madurez (Bhatta et al. 2007). Existen estudios donde se ha descrito que son una excelente fuente de proteínas, fibra, azúcares, incluso compuestos bioquímicos (alcaloides, taninos, terpenos, y flavonoides) por lo que puede ser considerado como una opción para la alimentación rumiantes (Talpada et al. 2002, Pereira et al. 2013, Rodríguez et al, 2018). Así mismo, se ha recomendado como complemento en proporciones de hasta el $40 \%$ de la dieta; sin embargo, los aportes nutricionales dependen del uso de las hojas o de las vainas por lo que los resultados en la alimentación animal son diversos (Sawal et al. 2004, Santos et al. 2015, Ruiz et al. 2020). Aunque existe información de la inclusión de las vainas de mezquite en dietas para animales, es poca la relacionada con sus características nutricionales y su degradación a nivel ruminal en distintas etapas de madurez. Por lo anterior, el objetivo del estudio fue conocer la composición química y la degradación ruminal de la vaina de mezquite en diferentes estados de madurez, por su importancia como alternativa en la alimentación de los rumiantes en las regiones áridas y semiáridas.

\section{MATERIALES Y MÉTODOS}

\section{Localización del experimento}

La fase experimental se realizó en el Rancho Dos Pivotes ubicado al Suroeste del Municipio de Zapotlán El Grande, del Estado de Jalisco, México; con coordenadas geográficas de $19^{\circ} 27^{\prime} 13^{\prime \prime} \mathrm{LN}$ y $103^{\circ} 27^{\prime} 57^{\prime \prime}$ LO, con una altitud de 1520 metros sobre el nivel del mar. El clima es semicálido, clasificado por Köppen en la modificación de García (2004) como (A)c (w0) (w) a (i') con una precipitación pluvial anual de $732 \mathrm{~mm}$ distribuidos en los meses de Junio a Septiembre, lluvias invernales o cabañuelas de manera ocasional. Su temperatura media es de 20.2 ${ }^{\circ} \mathrm{C}$. Las determinaciones químicas de las vainas de mezquite se realizaron en el Laboratorio de Nutrición del Centro Universitario del Sur de la Universidad de Guadalajara.

\section{Colecta y preparación de las muestras}

Las vainas se recolectaron en el municipio de Usmajac, Jalisco, México, de 15 árboles al azar y se recolectó de cada uno de ellos aproximadamente un kilogramo por separado de cada estado de madurez de la vaina indicado por su color y con base a las características físicas de la vaina (Pinzón et al. 2007). Los materiales experimentales de los estados de maduración vaina tierna y madura fueron colectados directamente del árbol, mientras que el material de vaina seca fue colectado de los frutos que se encontraban en suelo, teniendo así, tres tratamientos para ser evaluadas mediante cuatro repeticiones, muestras que se llevaron al laboratorio 
de nutrición animal para su proceso. Con la finalidad de obtener información de la composición química y degradabilidad ruminal de la vaina del mezquite, se evaluaron tres estados de maduración: a) vaina tierna (VT); de coloración verde fuerte y con semillas aun en formación; b) vaina madura (VM); de coloración morado intenso con líneas verdes y textura blanda; y c) vaina seca (VS); coloración morado pálido y con las semillas completamente formadas, una vaina gruesa y textura dura.

\section{Análisis químico}

Todas las vainas fueron secadas en una estufa a $55{ }^{\circ} \mathrm{C}$ por $48 \mathrm{~h}$ y posteriormente molidas en un molino de martillos con criba de $2 \mathrm{~mm}$ para su posterior análisis. A cada muestra se le determinó por cuadruplicado la materia seca total (MST), proteína cruda (PC) mediante el método Kjeldahl, extracto etéreo (EE) por el método Soxhlet, cenizas por combustión en mufla a $600{ }^{\circ} \mathrm{C}(\mathrm{C})$ y materia orgánica (MO) por diferencia, todos mediante la técnica descritas por la AOAC (2012). La determinación de las fracciones de fibra (FDN y FDA) se realizaron secuenciales y por cuadruplicado por el método de Van Soest et al. (1991). Se determinó el contenido de carbohidratos no fibrosos (CNF) mediante la ecuación [100 - (FDN, $\%+\mathrm{PC}, \%+\mathrm{EE}, \%,+\mathrm{C}, \%)]$ de acuerdo con Weiss (1999), sin corrección de proteína. La energía metabolizable (EM) por kilogramo de materia seca fue calculado de acuerdo con Weiss (1993).

\section{Degradabilidad in situ}

Para determinar la DIS de la MS de las tres etapas de maduración del fruto del mezquite, de acuerdo con la metodología propuesta por Vanzant et al. (1998); se utilizaron dos novillos Holstein con peso vivo de $350 \pm 43 \mathrm{~kg}$, fistulados con cánula ruminal permanente de $10 \mathrm{~cm}$ de diámetro central (Bar Diamond Lane, Parma, ID, USA), que fueron alimentados con $70 \%$ de heno de alfalfa y $30 \%$ de concentrado comercial al $12 \%$ de PC durante todo el periodo experimental. Se utilizaron bolsas de poliseda de 10 $x 15 \mathrm{~cm}$ con un tamaño de poro de $52 \pm 10 \mu \mathrm{m}$ previamente identificadas, en las cuales se depositaron $5 \mathrm{~g}$ de muestra. Las bolsas se cerraron con ligas de hule, y después se sujetaron a una cadena utilizando hilo de nylon. Se incubaron en el rumen por cuadriplicado para cada tratamiento por 16, 24, 36, 48, 72 y 96 h; también se colocaron bolsas testigo (blancos) sin muestra, en cada tiempo de incubación. Las bolsas fueron depositadas secuencialmente, con el fin de que todas las bolsas fueran removidas al mismo tiempo; posteriormente se lavaron con agua potable hasta que el agua de lavado saliera clara. Se dejaron secar a temperatura ambiente y después en estufa de aire forzado a $55{ }^{\circ} \mathrm{C}$ por $48 \mathrm{~h}$; posteriormente se registró su peso y se determinó la DIS de la MS, y del residual posterior a la digestión ruminal se determinó la concentración de cenizas para determinar la degradabilidad de la materia orgánica (AOAC 2012).

\section{Análisis estadístico}

Los datos obtenidos de la composición química y la degradabilidad in situ fueron analizados mediante un diseño completamente al azar con cuatro repeticiones por tratamiento. Se utilizó el procedimiento PROC GLM del paquete estadístico SAS, 2009 y comparación de medias mediante la prueba de Tukey (SAS 2006). La significancia se declaró a $\mathrm{P}<0.05$. El modelo estadístico utilizado fue:

$$
Y_{i j}=\mu+T_{i}+e_{i j}
$$

Donde $\mathrm{Y}_{i j}$ es la variable de respuesta; $\mu$ es la media general; $\mathrm{T}_{i}$ el efecto del i-ésimo tratamiento; e es el error experimental.

\section{RESULTADOS}

Se encontraron diferencias $(P<0.05)$ en la composición química entre los distintos estados de madurez de la vaina (Tabla 1). Las vainas tiernas, tuvieron el valor más alto de PC (18.3\%), el cual disminuyó a $12.30 \%$ en las vainas maduras y a $13.44 \%$ en vainas secas. El valor más alto de FDN se observó en las vainas tiernas (47.62\%), y disminuyó a 27.74 y $26.70 \%$ en las vainas maduras y secas de manera respectiva. El contenido de carbohidratos no fibrosos fue mayor en las vainas secas $(53.50 \%)$, seguido de 
Tabla 1. Composición química de las vainas de mezquite en tres estadios de maduración (\%).

\begin{tabular}{lccc}
\hline & \multicolumn{3}{c}{ Tratamientos } \\
\cline { 2 - 4 } Componentes & Vaina Tierna & Vaina Madura & Vaina Seca \\
\cline { 2 - 4 } Materia Seca & $33.33^{b}$ & $37.13^{b}$ & $80.48^{a}$ \\
Materia Orgánica & $86.12^{b}$ & $89.58^{b}$ & $96.23^{a}$ \\
Proteína Cruda & $18.13^{a}$ & $12.30^{b}$ & $13.44^{b}$ \\
Extracto etéreo & $7.21^{a}$ & $7.53^{a}$ & $2.59^{b}$ \\
Fibra detergente neutro & $47.62^{a}$ & $27.74^{b}$ & $26.70^{b}$ \\
Fibra detergente ácido & $31.66^{a}$ & $20.31^{b}$ & $10.64^{c}$ \\
Hemicelulosa & $15.96^{a}$ & $7.43^{c}$ & $16.06^{a}$ \\
Cenizas & $13.88^{a}$ & $10.42^{b}$ & $3.77^{c}$ \\
Carbohidratos no fibrosos & $13.16^{c}$ & $42.01^{b}$ & $53.50^{a}$ \\
Energía Metabolizable (Mcal/kg MS) & $2.43^{c}$ & $2.86^{b}$ & $3.03^{a}$ \\
\hline$a, b$ Valores en las medidas en el mismo renglón con distinta literal son diferentes (P< \\
0.05).
\end{tabular}

las vainas maduras (42.01\%), y el valor más bajo se observó en las vainas tiernas (13.16\%). De igual manera, el contenido de energía metabolizable fue mayor en las vainas secas y menor en la vaina tierna.

En relación con el coeficiente de degradabilidad in situ de la materia seca de las vainas de mezquite (Tabla 2$)$, fue menor $(P<0.05)$ en VT en todas las horas de incubación, y fue similar entre VM y VS $(P>0.05)$ en todas las horas de incubación, excepto a la hora 96, donde VS fue mayor $(P<0.05)$. Por otra parte, la DIS de la MO (Tabla 3), fue diferente entre los tratamientos $(P<0.05)$ hasta las primeras $36 \mathrm{~h}$, los valores más altos se encontraron en VT y $\mathrm{VM}$, posterior a esta hora no se encontraron diferencias entre ellos $(P>0.05)$.

\section{DISCUSIÓN}

En estudios previos de composición química de vainas de mezquite, se reportan valores de PC de $11.20 \%$ en vainas tiernas, por debajo de lo encontrado en este estudio (18.13\%) y de 10.28 a $12.05 \%$ en maduras de Prosopis laevigata y Prosopis spp. (Álvarez et al. 2015, Armijo et al. 2019, Gárcia et al. 2019), valores similares a los encontrados en este estudio. Sin embargo, en otros estudios reportan valores distintos de PC de entre 7.0 y $18.5 \%$ (Koech et al. 2010, Girma et al. 2011), 17.8\% en P. velutina (Rodríguez et al. 2015), variaciones que se pueden a- tribuir a las diferentes especies y también, aunque no se mencionan a los distintos estados de madurez de las vainas usadas en estos estudios.

El contenido promedio de FDN de vainas de Prosopis spp. reportado en estudios previos es de 20 a 34\%, (Ali et al. 2012, Baraza et al. 2008, Romero y Urrutia 2004) y de 23.92 a $29.10 \%$ en vainas maduras de P. laevigata (Álvarez et al. 2015, García et al. 2019), las cuales son similares a los reportados en la vainas maduras y secas en este estudio. En el caso de las vainas secas son las que presentan mayor contenido de carbohidratos no fibrosos, en otros estudios se reporta $49.20 \%$ de carbohidratos no fibrosos entre 48.1 y $49.20 \%$ (Rodríguez et al. 2015, García et al. 2019). Entre los compuestos presentes en la fracción de carbohidratos no fibrosos se encuentran azúcares, almidones, ácidos orgánicos, fructanos y pectinas que proporcionan energía, por lo que representan una fuente de energía en la dieta de los rumiantes (Peña et al. 2016). La composición química de las vainas de mezquite puede variar por factores como la madurez de la vaina, la especie o variedad, el clima y el suelo (Bhatta et al. 2007, Mellado 2016, Ruiz 2011). El alto contenido de carbohidratos no fibrosos, el aporte adecuado de PC y el bajo contenido de FDN en las vainas de Prosopis spp. constituyen una buena fuente de energía para los rumiantes (González et al. 2008, Ríos et al. 2012); el valor de energía reportado en vainas tiernas es de $1.650 \mathrm{Mcal} \mathrm{kg} \mathrm{MS}^{-1}$ y $1.482 \mathrm{Mcal} \mathrm{kg} \mathrm{MS}^{-1}$ en vainas 
Tabla 2. Coeficiente de digestibilidad in situ de la materia seca del mezquite en tres estadios de maduración (\%)

\begin{tabular}{ccccc}
\hline & \multicolumn{3}{c}{ Tratamientos } \\
\cline { 1 - 3 } $\begin{array}{c}\text { Horas } \\
\text { Incubación }\end{array}$ & Vaina Tierna & Vaina Madura & Vaina Seca & \multirow{2}{*}{ EEM $^{1}$} \\
\cline { 2 - 4 } 96 & $65.35 \mathrm{c}$ & $73.64^{b}$ & $78.70^{a}$ & 0.95 \\
72 & $66.89^{b}$ & $73.62^{a}$ & $74.86^{a}$ & 1.42 \\
48 & $69.77^{b}$ & $78.74^{a}$ & $77.34^{a}$ & 2.53 \\
36 & $60.82^{b}$ & $74.68^{a}$ & $76.13^{a}$ & 1.97 \\
24 & $61.29^{b}$ & $70.49^{a}$ & $70.64^{a}$ & 1.40 \\
16 & $53.53^{b}$ & $67.26^{a}$ & $69.95^{a}$ & 3.13 \\
\hline
\end{tabular}

$a, b$ Valores en las medidas en el mismo renglón con distinta literal son diferentes $(\mathrm{P}<0.05) .{ }^{1}$ EEM: Error estándar de la media

Tabla 3. Coeficiente de digestibilidad in situ (\%) de la materia orgánica del mezquite en tres estadios de maduración.

\begin{tabular}{|c|c|c|c|c|}
\hline \multicolumn{5}{|c|}{ Tratamientos $^{1}$} \\
\hline Horas & Vaina Tierna & Vaina Madura & Vaina Seca & \multirow[b]{2}{*}{$\mathrm{EEM}^{2}$} \\
\hline Incubación & \multicolumn{3}{|c|}{$\%$} & \\
\hline 96 & $78.17^{a}$ & $81.46^{a}$ & $80.60^{a}$ & 0.85 \\
\hline 72 & $79.89^{a}$ & $82.02^{a}$ & $77.96^{a}$ & 1.07 \\
\hline 48 & $84.58^{a}$ & $86.33^{a}$ & $83.55^{a}$ & 2.12 \\
\hline 36 & $82.61^{b}$ & $85.13^{a}$ & $75.48^{c}$ & 1.75 \\
\hline 24 & $85.02^{a}$ & $84.66^{a}$ & $81.62^{b}$ & 0.58 \\
\hline 16 & $81.00^{a b}$ & $83.43^{a}$ & $73.93^{b}$ & 1.74 \\
\hline
\end{tabular}

maduras (Armijo et al. 2019), lo cual contrasta con los calores encontrados en el presente estudio, posiblemente esta variación se deba a la edad o estado de madurez de las vainas al momento de la cosecha, el suelo y clima, la especie o variedad, la ubicación e incluso los factores antinutricionales en las semillas que pueden influir el valor nutritivo de los vainas (King' ori et al. 2011, Chatuverdi y Sahoo 2013).

Los resultados indican que disminuye el contenido de PC y FDN, mientras que el contenido de carbohidratos solubles aumenta debido posiblemente a la madurez en las vainas de mezquite. Los valores observados de FDN y FDA indican bajo contenido de fibra indigestible en las vainas maduras y secas. Este indicador tiene importancia para definir el estado de madurez al que deben ser cosechadas las vainas de mezquite, dependiendo de las necesidades de forraje de los animales.

El mayor coeficiente de degradabilidad in situ de la materia seca observado en los tratamientos VM y VS puede ser explicado por el menor contenido de FDA y FDA, debido a que un mayor contenido de ambos se asocia con menor digestibilidad (Figuerido et al. 2007). Sawal et al. (2004) mencionan que las vainas de mezquite presentan altos coeficientes de digestibilidad de la MS (71.10 a $82.56 \%)$, lo cual coincide con lo encontrado en este estudio en las vainas maduras y secas, que fue de 73.64 y $78.70 \%$ de manera respectiva, y menor en las vainas tiernas (65.35\%). La digestibilidad de la MS de las vainas de Prosopis spp. reportada por diversos autores es variable; en vainas tiernas de Prosopis spp. fue de $66.99 \%$ y en vainas secas de $64.81 \%$ (Armijo et al. 2019), ambos valores son similares a lo observado en VT y menores que en VM y VS; Barros y Filho (1986) reportan valores de $82.6 \%$ en vainas maduras de Prosopis juliflora. El valor nutricional de las vainas de mezquite varía de acuerdo a su estado madurez, por lo que considerarla es muy importante, ya que puede ser un elemento a considerar al utilizarlas como ingrediente o sustitutos de ingredientes en las dietas integrales, particularmente en tiempo de estiaje, ya que puede ayudar reducir los gastos de mantenimiento en las explotaciones pecuarias. 


\section{CONCLUSIONES}

Las vainas maduras y secas de mezquite presentaron los resultados más bajos de FDN, FDA, y los valores más altos en carbohidratos no estructurales, degradabilidad in situ de la materia seca y orgánica, pero, su contenido de PC fue menor, en comparación con las vainas tiernas. Estos resultados sugieren que el estado de madurez de la vaina debe ser considerado para la utilización de estas en la alimentación animal, dada la relación que existe entre el estado de madurez y su contenido nutricional que está relacionado con óptimo de aprovechamiento de las vainas de mezquite por el animal.

\section{LITERATURA CITADA}

Ali AS, Tudsri S, Rungmekarat S, Kaewtrakulpong K (2012) Effect of feeding Prosopis spp. pods and leaves on performance and carcass characteristics of Afar sheep. Kasetsart Journal of Natural Science 46: 871-881.

Álvarez FG, García LJC, Pinos RJM, Jasso PY, Tristaán PFM, González GR (2015) Maguey (Agave spp.) silage production with either alfalfa or mesquite pod meal as protein sources. Journal of Animal and Plant Sciences 24: 3714-3721.

Andrade MHM, Córdova TAV, García GT, Kawas JR (2011) Alternative foods for small ruminants in semiarid zones, the case of mesquite (Prosopis laevigata spp.) and nopal (Opuntia spp.). Small Ruminant Research 98: 83-92.

AOAC (2012) Official Methods of Analysis 19th ed. Association of Official Analytical Chemists: Gaithersburg, Mary Land, USA. 771p.

Armijo NMG, Moreno RA, Blanco CE, Borroel GVJ, Reye CJL (2019) Vaina de mesquite (Prosopis spp.) alimento para el Ganado caprino en el semidesierto. Revista Mexicana de Ciencias Agrícolas 10: 113-122.

Bhatta R, Vaithiyanathan S, Singh NP, Verma DL (2007) Effect of feeding complete diets containing graded levels of Prosopis cineraria leaves on feed intake, nutrient utilization and rumen fermentation in lambs and kids. Small Ruminant Research 67: 75-83.

Baraza E, Ángeles S, García A, Valiente A (2008) Nuevos recursos naturales como complemento de la dieta de caprinos durante la época de seca en el Valle de Tehuacán, Puebla. Interciencia 33: 891-896.

Barros NAM, Filho TL (1986) Effect of progressive replacement of molasses by Prosopis juliflora (SW) D. C. pods in ruminant rations. The current state of knowledge on Prosopis juliflora. II International Conference of Prosopis. Recife, Brasil. pp. 349-359.

Carrillo F, Gómez L, Arreola A (2014) Efecto de la poda sobre potencial productive de mezquitales nativos en la comarca lagunera, México. Revista Chapingo. Serie Zonas Áridas. 6: 47-54.

Chatuverdi OH, Sahoo A (2013) Nutrient utilization and rumen metabolism in sheep fed Prosopis spp. pods and Cenchrus grass. Springer Plus 2: 1-7.

Figuerido MP, Cruz PG, Costa SS (2007) Fraccionamiento dos carbohidratos e componentes nitrogenados do farelo e diferentes partes integrantes da vagem de algaroba (Prosopis juliflora (Swartz) D. C.) Revista Brasileira Saúde Producao Animal 8: 24-31.

García E (2004) Modificaciones al Sistema de Clasificación Climática de Köppen (adaptarlo a las condiciones de la República Mexicana. $5^{\mathrm{a}}$ ed. Instituto de Geografía, UNAM. México. $91 \mathrm{p}$.

García, LJC, Durán GHM, de Nova JA, Álvarez FG, Pinos RJM, Lee RH, López AS, Ruiz TD, Rendón, HJA, Vicente MJC, Salina RM (2019) Producción y contenido nutrimental de vainas de tres variantes de mezquite (Prosopis laevigata) en el altiplano potosino, México. Agrociencia 53: 821-831. 
Girma M. Urge M, Animut G (2011) Ground Prosopis spp. pods as feed ingredient in poultry diet: effects on growth and carcass characteristics of broilers. International Journal of Poultry Science 10: 970-976.

Gómez A, Pinos J, Aguirre J (2009) Manual de producción caprina. Primera edición. Ediciones Universidad Autónoma de San Luis Potosí. México. 186p.

González YH, Ortega JA, Rodríguez RA, Aguirre CO, Gurrola AG, Macías RM (2008) Métodos inductivos para maximizar la germinación de semilla de germoplasma nativo en vivero para sistemas silvopastoriles en Nayarit, México. Zootecnia Tropical 26: 355-358.

Khobondo JO 2019 Effect of incorporation of ground Prosopis juliflora pods in layer diet on weight gain egg production, and natural antibody titer in KALRO genetically improved indigenous chicken. Tropical Animal Health and Production. 51: 2213-2218.

King'ori AM, Odero WJA, Guliye A (2011) Mathenge (Prosopis spp.): an underutilized livestock feed resource in Kenya. Research Journal of Animal Science 5: 43-51.

Koech OK, Kinutia RN, Wahome RG, Choge SK (2010) Effects of Prosopis spp. seedpod meal supplement on weght gain of weaner Galla goats in Kenya. Research Journal of Animal Science 4: 58-62.

López FYL, Goycoolea MF, Valdez AM, Calderón AM (2006) Goma de mezquite: una alternativa de uso industrial Interciencia 31: 183-189.

Mellado M (2016) Dietary selection by goats and the implications for range management in the Chihuahuan desert: a review. The Rangeland Journal 38: 331-341.

Peña ALV, Pinos RJM, Juárez FB, Yañez EL (2016) Effects of Prosopis laevigata pods on growth performance, ruminal fermentation and blood metabolites in finishing lambs. South African Journal of Animal Science 46: 360-365.

Pereira TCJ, Albuquerque PML, Santana OLA, da Silva AC, de Oliveira SHG, dos Santos PM, Presidio APJ, Batista SA (2013) Mesquite pod meal in diet for lacting goats. Revista Brasileira de Zootecnia 42: 102-108.

Pinzón PIG, Fischer G, Corredor G (2007) Determinación de los estados de madurez del fruto de gulupa (Passiflora edulis Sims.). Agronomía Colombiana 25: 83-95.

Ríos SJC, Valenzuela NLM, Rivera GM, Trucios CR, Sosa PG (2012) Diseño de un Sistema silvopastoril en zonas degradadas con mezquite en Chihuahua, México. Tecnociencia Chihuahua 6: 174-180.

Rodríguez RFA, Rendón HJA, Álvarez FG, García LJC (2018) Digestibilidad in vitro de una ración a base de vaina de mezquite, ensilado de maguey y nopal para la producción de leche en cabras. In: Herrera JC, Chay CAJ, Casanova LF, Piñeiro VAT, Márquez BL, Santillán FE, Menocal AJ (eds). Avances de la investigación sobre producción animal y seguridad alimentaria en México. 1a Ed. Morelia Michoacán, México. pp: 533-538.

Romero J, Urrutia J (2004) Suplementación con costilla de vaca y vaina de mezquite a cabras de lactancia. Folleto técnico número 16 INIFAP. Centro de Investigación Regional del Noreste. México. 2p. http://inifap cirne.gob.mx/Biblioteca/Publicaciones/69.pdf. Fecha de consulta: 5 de febrero de 2021.

Ruiz NJE, Hernández RJ, Hernández MJ, Mendoza CJ, Abraham JM, Isiordi LPM, Mireles AAI (2020) Mesquite (Prosopis spp.) tree as a feed resource for animal growth. Agroforestry Systems 94: 1139-1149

Santos EJ, Albuquerque PML, Presídio APJ, Vitória MJ, Santos SAC, Ramos PCA 2015 Mesquite pod meal in sheep diet: intake, apparent digestibility of nutrients and nitrogen balance. Acta Scientiarum. Animal Science 37: 55-59. 
Sawal RK, Ratan R, Yadav BS (2004) Mesquite (Prosopis juliflora) pods as a feed resource for livestock. A review. Asian-Australian Journal of Animal of Science 17: 719-725.

SAS (2006). User's guide. Statistical Analysis System Institute Inc. Version 9.1.3 Cary, NC, USA. 44p.

Talpada PM, Pendya PR, Patel GR, Patel DC, Desai M (2002) Utilization of complete feed using Prosopis juliflora pods as a ration of growing crossbred calves. Indian Journal of Animal Nutrition 19: 1-6.

Tawel HZ, Tas BM, Smith HJ, Elgersma A, Dijkstra J, Tamminga S (2005) Effects of feeding perennial ryegrass with an elevated on intake, rumen function and performance of dairy cows. Animal Feed Science and Technology 121: 243-256.

Van Soest PJ, Robertson B, Lewis BA (1991) Methods for dietary fiber, neutral detergent fiber and nonstarch polysaccharides in relation to animal nutrition. Symposium: carbohydrate methodology, metabolism, and nutritional implications in dairy cattle. Journal of Dairy Science 74: 3583-3597.

Weiss WP (1993) Predicting energy values of feeds. Journal of Dairy Science 76: 1802-1811.

Weiss WP (1999) Energy prediction equations for ruminant feeds. In: Proceedings of the Cornell Nutrition Conference for Feed Manufacturers. New York State College of Agriculture and Life Sciences, Cornell University, USA. pp: 176-185.

Xu BC, Gichuki P, Shan L, Li FM (2006) Aboveground biomass production and soil water dynamics of four leguminous forages in semiarid region northwest China. South African Journal of Botany 72: 507-516.

Vanzant ES, Cochran RC, Titgemeyer EC (1998) Standardization of in situ techniques for ruminant feedstuff evaluation Journal of Animal Science 76: 2717-2729 